\title{
Numerical Simulation of Thrombolysis in Robot-Assisted Retinal Vein Cannulation
}

\author{
Shunlei Li, ${ }^{1}$ Jiawen Pan, ${ }^{2,3}$ Juan Ji $\mathbb{C}^{4},{ }^{4}$ Guanghang Wang, ${ }^{3}$ and Baobao Qi ${ }^{1}$ \\ ${ }^{1}$ Beijing Key Laboratory of Advanced Manufacturing Technology, Beijing University of Technology, Beijing 100124, China \\ ${ }^{2}$ Faculty of Information Engineering and Automation, Kunming University of Science and Technology, Kunming 650031, China \\ ${ }^{3}$ College of Future Technology, University of Chinese Academy of Sciences, Beijing 100049, China \\ ${ }^{4}$ Department of Pathology, Sichuan Cancer Hospital \& Institute, Sichuan Cancer Center, School of Medicine, \\ University of Electronic Science and Technology of China, Chengdu 610041, China
}

Correspondence should be addressed to Juan Ji; juan_j2018@163.com

Received 20 August 2020; Revised 26 October 2020; Accepted 11 November 2020; Published 27 November 2020

Academic Editor: M. Irfan Uddin

Copyright (c) 2020 Shunlei Li et al. This is an open access article distributed under the Creative Commons Attribution License, which permits unrestricted use, distribution, and reproduction in any medium, provided the original work is properly cited.

\begin{abstract}
Robot-assisted retinal cannulation is an eye surgical procedure which can dissolve the obstruction by using robot to inject anticoagulant into occluded vessel. The current research on the critical parameters of cannulation for human is scarce because of the immature technology. Considering the influence of microneedle, this work investigated the effects of drug concentration, injection velocity, injection position, and size of clot on cannulation by theoretical analysis and finite element analysis. For finite element analysis, the multiphysics continuum model was established to demonstrate species transport and reaction which simulates the entire lytic process of the occlusive clot, and four cell zones were established to describe the generation of plasmin (PLS) with the addition of tissue-type plasminogen activator (tPA) and fibrinolysis of clot by importing subroutines into each cell zone under the conditions of constant clot size and variable size, respectively. The results imply that the most efficient value of tPA concentration is $50 \mathrm{nM}$, injection velocity is $60 \mathrm{~mm} / \mathrm{s}$ for clot length of $0.1 \mathrm{~mm}$, and the best position to insert the cannula is $0.5 \mathrm{~mm}$ in front of the thrombus. For different clot lengths of $0.1 \mathrm{~mm}$ to $0.6 \mathrm{~mm}$, the optimal range of tPA concentration and injection velocity is from $20 \mathrm{nM}$ to $70 \mathrm{nM}$ and from $40 \mathrm{~mm} / \mathrm{s}$ to $60 \mathrm{~mm} / \mathrm{s}$, respectively, and explores the reasonable injection position of $0.3 \mathrm{~mm}$ to $0.5 \mathrm{~mm}$ in front of clot in a vein of $100 \mu \mathrm{m}$. This conclusion can be used to perform robot-assisted cannulation surgery to improve fibrinolytic efficiency.
\end{abstract}

\section{Introduction}

Retinal vein occlusion (RVO) is one of the most common retinal disease where thrombus inside retinal veins distort the delivery of oxygen, resulting in central retinal vein occlusion (CRVO) and branch retinal vein occlusion (BRVO) [1-5]. It impairs the vision of an estimated 16.4 million people in the world [1]. Up to present, the therapeutic strategy for retinal vein occlusion has aimed to limit the damage caused by the obstruction, but cannot dissolve the occlusion in vessel. Consequently, RVO patients need to be treated on a periodic basis with costly therapies to offer limited symptom relief $[6,7]$, such as intravitreal injections [8] and laser photocoagulation [9]. A promising potentially curative treatment method is retinal vein cannulation, also known as retinal endovascular surgery (REVS), as shown in Figure 1. By injecting an anticoagulant directly into the occluded vessel, REVS is focused on thrombolysis to cure RVO completely [10]. Cannulation of the involved vessel and removal of the clot may provide a more permanent solution with a less demanding follow-up [11].

Because of the small size of retinal vessel, surgical robot and machine vision detection [12] are essential for cannulation. Smet et al. injected ocriplasmin into porcine eyes to clear clot by robot-assisted cannulation [13], and Willekens et al. performed cannulation to release drugs for more than $3 \mathrm{~min}$ and dissolved clot in 15 of 18 porcine eyes with the help of surgical robot [14]. Their research is limited to animal 


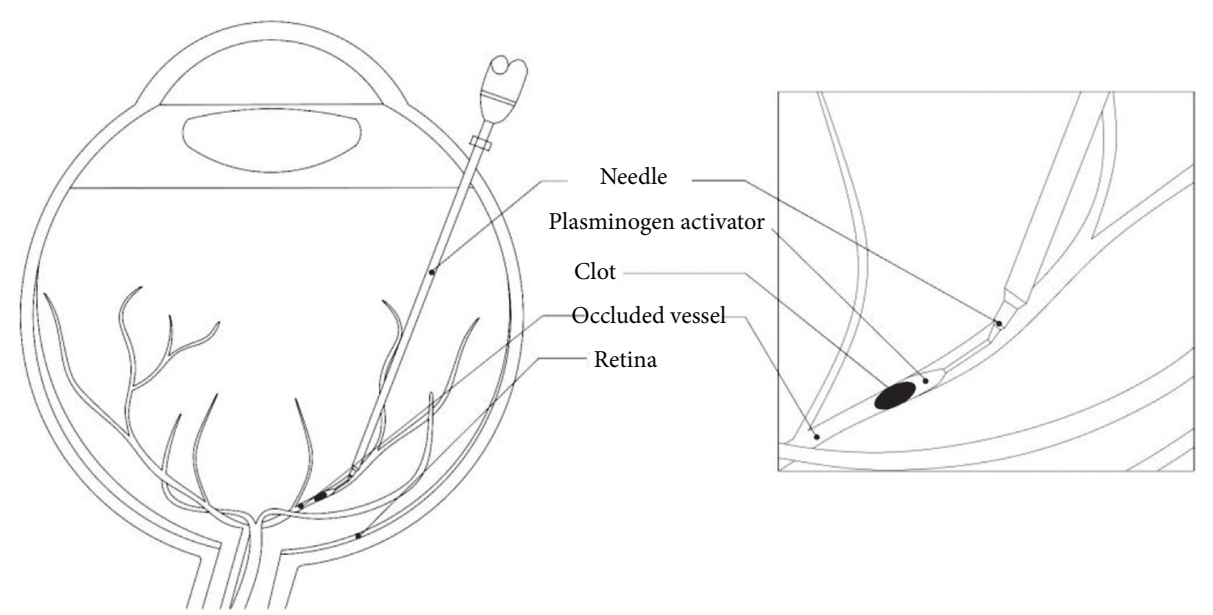

FIgURE 1: Retinal vessel cannulation to dissolve retinal vessel obstruction.

experiments. However, the shape and size of porcine eyes are different from those of human eyes. Gijbels et al. successfully cured four patients with robot devices as the first robotassisted retinal rein cannulation in the world, but they did not focus on the details during surgery process, such as injection velocity, drug concentration, injection position, and variable size of clot [15]. Thus, cannulation is still immature and dangerous for smaller vessel. Due to the lack of human operation and experimental data, the environment and important parameters of cannulation remain unclear.

Some models were established to investigate the process of fibrinolysis. Scott L. Diamond et al. modeled clot lysis by solving a set of reaction-diffusion-convection equations which contains no adjustable parameters [16]. Piebalgs et al. developed a multiphysics model for the dissolution of fibrin clots to obtain the shape and subsequent lysis with three different pressure drops [17]. Wootton et al. incorporated the effect of outer convection by solving the continuity and Navier-Stokes equations for blood flow [19]. Recently, stochastic models have been suggested as a method to analyse the effects of clot structure on thrombolysis [20, 21]. In addition, Rijken et al. used a purified experiment system to compare the fibrinolytic properties of two molecular forms of tPA [22], and Anand et al. used a numerical model to predict lysis fronts moving across biogels [27]. However, these methods did not consider the critical parameters of cannulation.

This study aims to detect the influence of each parameter, such as injection velocity, drug concentration, and injection position, on the thrombolysis process in robotassisted surgery by theoretical simulation and fluid calculation of finite element. In addition, this study considers the influence of microneedle and variable clot size in this model and cannula into the thrombus when inadvertent operation occurs. The results obtained from this work will be used to optimize the operation process with robot devices in the near future.

\section{Materials and Methods}

2.1. Geometry of the Model. As shown in Figure 2, the computational geometry is a vein with occlusion where the inner diameter is chosen to be $100 \mu \mathrm{m}$ in accordance with the dimensions of the human retinal vein [23] and the distance from blood vessel inlet to outlet is long enough to reduce the influence of artificial boundary conditions on flow and protein concentration. Additionally, the length of clot is chosen to be from $0.1 \mathrm{~mm}$ to $0.6 \mathrm{~mm}$ in this simulation. Finally, the implement for cannulation is a glass microneedle that has $500 \mu \mathrm{m}$ long tip being angulated $30^{\circ}$ with respect to the shaft and the diameter of $30 \mu \mathrm{m}$ (Clunbury Scientific, LLC, BloomfieldHills (MI), USA) [7].

\subsection{Reaction Kinetics and Transport Equations of Species.} Figure 3 shows the reaction kinetics of thrombolysis that plasminogen (PLG) is activated by tissue-type plasminogen activator (tPA) absorbed on fibrin surface (bound phase) to form plasmin (PLS), which can degrade the fibrin mesh that stabilizes blood clots. $\alpha_{\mathrm{f}}$ specie $\alpha$ (tPA, PLS, PLG) in free phase, $\alpha_{b}$ is specie $\alpha$ in bound phase, PLS AP $_{\text {PLS which }}$ cannot dissolve the clot due to inhabitation of AP. Clot consists of a numbers of protofibril which can be degraded by PLS. Bound phase is available to absorb protease on fibrin surface. In order to evaluate the temporal and spatial changes in concentration, this method describes transport equations in the free phase and bound phase.

In order to improving the accuracy of the method, we considered the main inhibitory effect of $\alpha 2$-antiplasmin ( $\alpha 2$ AP) in free phase which can inhibit the fibrinolysis extremely [25] and be showed as follows:

$$
\frac{\mathrm{d}\left[C_{\mathrm{AP}} \varepsilon\right]}{\mathrm{d} t}=-k_{\mathrm{APC}_{\mathrm{AP}} C_{\mathrm{PLS}} \varepsilon}
$$




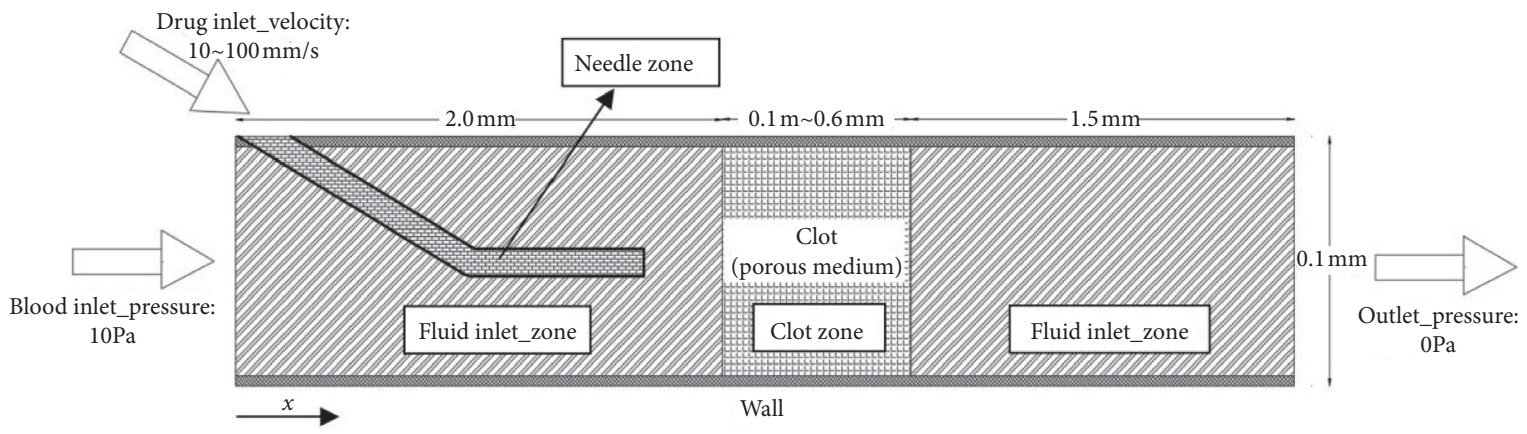

Figure 2: Thrombolytic simulation model, boundary conditions, and cell zones.

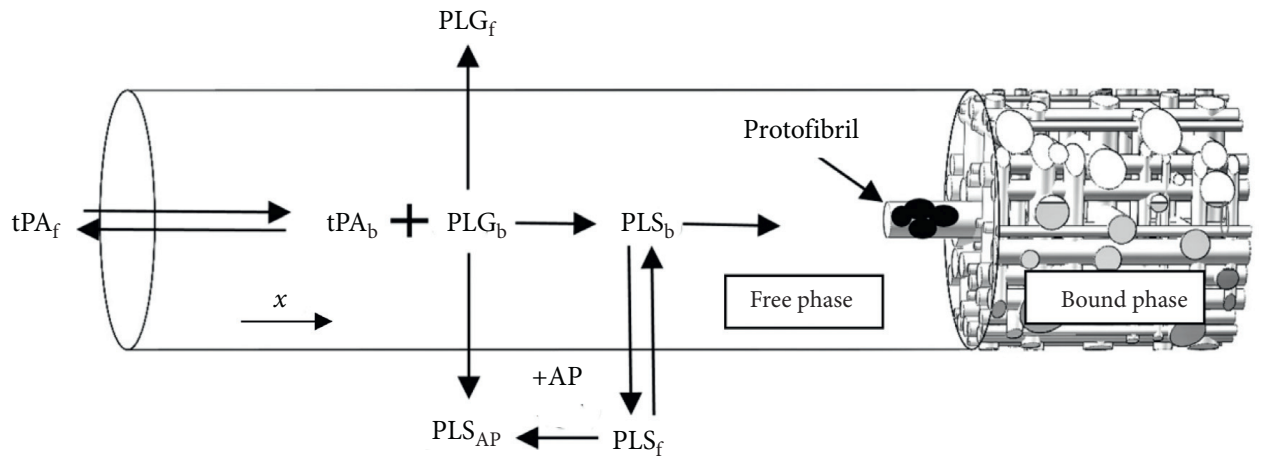

FIgURE 3: The process of thrombolysis.

TABle 1: Nomenclature.

\begin{tabular}{|c|c|c|c|c|}
\hline Symbol & Meaning & Value & Unit & Reference \\
\hline$\alpha_{0}$ & Amount of fibrin monomers per protofibril length & 0.0444 & $\mathrm{~nm}^{-1}$ & [16] \\
\hline$D$ & Dispersion coefficient & $5 \times 10^{-11}$ & $\mathrm{M}^{2} \mathrm{~s}^{-1}$ & [17] \\
\hline$k_{2}$ & Michaelis-Menten reaction coefficient & 15 & $\mathrm{~s}^{-1}$ & {$[16,34]$} \\
\hline$k_{\mathrm{AP}}$ & Antiplasmin reaction coefficient & 10 & $\mu \mathrm{M}^{-1} \mathrm{~s}^{-1}$ & [24] \\
\hline$k_{\text {ads,PLG }}$ & Forward adsorption coefficient of PLG & $1.087 \times 10^{-4}$ & $\mu \mathrm{M}^{-1} \mathrm{~s}^{-1}$ & {$[27]$} \\
\hline$k_{\text {ads,PLS }}$ & Forward adsorption coefficient of PLS & $0.500 \times 10^{-3}$ & $\mu \mathrm{M}^{-1} \mathrm{~s}^{-1}$ & {$[27]$} \\
\hline$k_{\mathrm{ads}, \mathrm{tPA}}$ & Forward adsorption coefficient of tPA & $1.148 \times 10^{-4}$ & $\mu \mathrm{M}^{-1} \mathrm{~s}^{-1}$ & [27] \\
\hline$k_{\text {cat }}$ & Lysis reaction rate coefficient & 10 & $\mathrm{~s}^{-1}$ & {$[16]$} \\
\hline$k_{\mathrm{M}}$ & Michaelis-Menten reaction coefficient & 0.13 & $\mu \mathrm{M}$ & {$[16]$} \\
\hline$k_{\text {rev,PLG }}$ & Reversible adsorption coefficient of PLG & $4.131 \times 10^{-3}$ & $\mathrm{~s}^{-1}$ & [27] \\
\hline$k_{\text {rev,PLS }}$ & Reversible adsorption coefficient of PLS & $5.435 \times 10^{-5}$ & $\mathrm{~s}^{-1}$ & {$[27]$} \\
\hline$k_{\text {rev, tPA }}$ & Reversible adsorption coefficient of tPA & $6.658 \times 10^{-5}$ & $\mathrm{~s}^{-1}$ & {$[27]$} \\
\hline$L_{\mathrm{pf}}$ & Total length of protofibrils & 3 & $\mathrm{~mm}$ & - \\
\hline$N_{\mathrm{AV}}$ & Avogadro's constant & $6.05 \times 10^{23}$ & $\mathrm{~mol}^{-1}$ & {$[17]$} \\
\hline$p_{0}$ & Amount of protofibril per fibre cross-sectional area & 0.01116 & $\mathrm{~nm}^{-2}$ & {$[16]$} \\
\hline$R_{\mathrm{f} 0}$ & Initial radius of fibrin fibre & 250 & $\mathrm{~nm}$ & {$[17]$} \\
\hline$\rho_{\text {fibre }}$ & Density of fibrin in fibre & 0.28 & $\mathrm{gml}^{-1}$ & {$[16]$} \\
\hline$\rho_{\beta}$ & Density of fibrin in clot & 0.003 & $\mathrm{gml}^{-1}$ & {$[17]$} \\
\hline$\gamma$ & Amount of cuts required by plasmin to cleave & 0.1 & - & {$[16]$} \\
\hline - & One unit of fibrin & - & - & - \\
\hline$r_{0}$ & Average radius of a protofibril & 2.39 & $\mathrm{~nm}$ & {$[16]$} \\
\hline
\end{tabular}


where $k_{\mathrm{AP}}$ is antiplasmin reaction coefficient listed in Table 1. Because of the weak effect, this method ignored the influence of $\alpha_{2}$-macroglobulin (MG) and PAI-1 [26]. For the free phase, the equation is written as follows:

$$
\begin{aligned}
\frac{\partial}{\partial t}\left[C_{\alpha} \varepsilon\right]+\frac{\partial}{\partial x_{i}}\left[u_{i} C_{\alpha} \varepsilon\right]= & \frac{\partial}{\partial x_{i}}\left[D_{\alpha, i j} \frac{\partial\left(C_{\alpha} \varepsilon\right)}{\partial x_{j}}\right]-(1-\varepsilon) \\
& \cdot\left[k_{\mathrm{ads}, \alpha} C_{\alpha}\left(\theta_{\alpha}-S_{\alpha}\right)-k_{\mathrm{rev}, \alpha} S_{\alpha}\right] \\
& +(1+K) S_{\alpha} \frac{\partial \varepsilon}{\partial t}+\frac{\mathrm{d}\left[C_{\mathrm{AP}} \varepsilon\right]}{\mathrm{d} t},
\end{aligned}
$$

where (1) term is the rate of change of the free phase, (2) term is the effect of convection on concentration in the free phase, (3) term is the effect of diffusion, (4) term is the rate of absorption in free phase, and (5) term is about the rate of generation of species $\alpha$ (tPA, PLS, PLG). $S_{\alpha}$ and $C_{\alpha}$ are the bound and free phase concentrations of species $\alpha$, respectively, $\theta_{\alpha}$ is the concentration of total species $\alpha$ that can be absorbed to the surface of fibrin, and $\varepsilon$ is porosity (voidage) of clot. These refer to spatial variations. $D_{\alpha, i j}$ is the hydrodynamic dispersion, $k_{\mathrm{ads}, \alpha}$ and $k_{\mathrm{rev}, \alpha}$, and $K$ is forward adsorption coefficient, reversible adsorption coefficient, and solubilization constant. These parameters are invariant constants listed in Table 1.

For the bound phase, the equation is written as follows:

$$
\begin{aligned}
\frac{\partial S_{\alpha}}{\partial t}+\frac{\partial\left(u_{i} S_{\alpha}\right)}{\partial t}= & \frac{\partial}{\partial x_{i}}\left[D_{\alpha, i j} \frac{\partial S_{\alpha}}{\partial x_{j}}\right] \\
& +\left[k_{\mathrm{ads}, \alpha} C_{\alpha}\left(\theta_{\alpha}-S_{\alpha}\right)-k_{\mathrm{rev}, \alpha} S_{\alpha}\right] \\
& -\frac{K}{(1-\varepsilon)} S_{\alpha} \frac{\partial \varepsilon}{\partial t},
\end{aligned}
$$

where (1) term is the rate of change of concentrations of specie $\alpha$ with effect of convection in bound phase, (2) term is the effect of diffusion, (3) term is the rate of absorption in free phase, and (4) term is about the rate of generation of specie $\alpha$.

The concentration of the total amount of binding sites that are sterically accessible to the proteins can be found by calculating the amount of binding sites per fibrin monomer and the total amount of these monomers in a protofibril:

$$
\theta_{\alpha}=\alpha_{0} L_{\mathrm{pf}} \chi q_{\alpha} \frac{1}{N_{\mathrm{AV}} U_{0}},
$$

where $\chi$ is the number of protofibrils that are sterically accessible, $q_{\alpha}$ is the number of binding sites in a fibrin monomer for species $\alpha$, and $U_{0}$ is volume of computational cell. In this study, we assume that all the protofibrils in the fibrin fiber are sterically accessible:

$$
\chi=\pi \rho_{0}\left(R_{f}+r_{0}\right)^{2},
$$

where $r_{0}$ is the average radius of a protofibril, which is shown in Table 1.

The transport of PLG in the bound phase should subtract the consumption forming PLS. On the contrary, the transport of PLS should plus the generation. The equation is written as follows:

$$
G_{\mathrm{PLS}}=R_{\mathrm{PLG}}=\frac{k_{2} S_{\mathrm{PLG}} S_{\mathrm{tPA}}}{k_{\mathrm{M}}+S_{\mathrm{PLG}}},
$$

where $R_{\mathrm{PLG}}$ is the reduction of PLG, $G_{\mathrm{PLS}}$ is generation of PLS, and $k_{2}$ and $k_{\mathrm{M}}$ are Michaelis-Menten reaction coefficients listed in Table 1.

2.3. Fibrinolysis of Clot. In Diamond SL and Anand S's work [16], this method describes the clot as fibrous porous medium composed of fibrin strands where the specific permeability and porosity is correlated with the fiber radius. Clot lysis was described as a homogeneous shrinking of the fiber radius over time [27]:

$$
R_{\mathrm{f}}=\sqrt{R_{f 0}^{2}-\frac{L N_{\mathrm{AV}}}{\pi p_{0}\left(L_{\mathrm{pf}} / U_{0}\right) \alpha_{0}}},
$$

where $R_{f}$ is the fiber radius, $L$ is the concentration of fibrin lysed, and $U_{0}$ is volume of computational cell. In addition, $R_{f 0}$ is initial radius of fibrin fiber, $N_{\mathrm{AV}}$ is Avogadro's constant, $p_{0}$ is amount of protofibril per fiber cross-sectional area, $L_{\mathrm{pf}}$ is total length of protofibrils, and $\alpha_{0}$ is amount of fibrin monomers per protofibril length, and these constants are shown in Table 1.

The cutting of the clot lysed is described as follows:

$$
\frac{\partial L}{\partial t}=\gamma k_{\mathrm{cat}} S_{\mathrm{PLS}}
$$

where $\gamma$ is the solubilization rate per number of cuts required by PLS to cleave one unit of fibrin and $k_{\text {cat }}$ is lysis reaction rate coefficient. These constants are shown in Table 1.

In this method, porosity $\varepsilon$ can present the decent of thrombolysis and is written as follows:

$$
\begin{aligned}
\varepsilon & =1-\frac{\pi R_{f 0}^{2} L_{\mathrm{pf}}}{U_{0}}+\frac{N_{\mathrm{AV}}}{p_{0} \alpha_{0}} \int \gamma k_{\mathrm{cat}} S_{P L S} \mathrm{~d} t, \\
\varepsilon_{0} & =1-\frac{\rho_{\beta}}{\rho_{\text {fibre }}},
\end{aligned}
$$

where $\varepsilon_{0}$ is initial value of voidage, $\rho_{\beta}$ and $\rho_{\text {fibre }}$ is the density of fibrin in clot and fiber, respectively. These constants are shown in Table 1. In subsequent comparisons, $\varepsilon$ is an important dissolution index. The clot lysis is completed when the porosity gets to one.

In order to obtain temporal changes in viscous resistance in porous zone, permeability of it needs to be taken:

$$
k=\lambda \frac{R_{f}^{2}}{(1-\varepsilon)^{n}},
$$

where $k$ is the permeability, $n$ is the permeability coefficient, and $\lambda$ is the permeability constant.

2.4. Governing Equations of Fluid Flow. In the study, we considered the influence of microneedle and described the blood flow through a porous medium as laminar and heat exchange is ignored [17]. The existence of viscous force leads to alternating vortex which affects flow velocity and pressure 
change extremely around microneedle. The time-dependent control equation is

$$
\begin{gathered}
\frac{\partial \varepsilon}{\partial t}+\frac{\partial u_{i}}{\partial x_{i}}=0 \\
\rho\left(\frac{\partial u_{i}}{\partial t}+u_{j} \frac{\partial u_{i}}{\partial x_{j}}\right)=-\frac{\partial p_{i}}{\partial x_{j}}+\mu \frac{\partial^{2} u_{i}}{\partial x_{j}^{2}}-\frac{\mu}{k} u_{i},
\end{gathered}
$$

where $\rho$ is blood density, $\mu$ is viscosity coefficient, $u$ is velocity, $p$ is pressure, and $k$ is permeability of porous medium [18]. We assume the blood flow as Newtonian and incompressible, and clot as isentropic and homogeneous.

2.5. Finite Element Analysis. This work established the multiphysics continuum model to demonstrate species transport and reaction which simulates the entire lytic process of the occlusive clot. The species reaction function was described by equations (1)-(10) and fluent movement was described by equations (11)-(13).

Source terms of tPA, PLG, and PLS implemented in ANSYS Fluent via user-defined functions (UDFs). This study set the blood flow as laminar flow and describes species transport by Species Transport Model where fibrinolysis of clot occurs in porous zone. The multiphysics continuum model was divided into four cell zones of fluid inlet, fluid outlet, needle zone, and clot zone, where subroutine of sources terms was imported as shown in Figure 2. For cell zone conditions, equations (9) and (13) were used to describe fluid porosity and viscous resistance of porous zone in cot zone, respectively. This model set equations (10) and (13), where $\varepsilon$ is equal to $\varepsilon_{0}$ as initialization, and equations (1)-(9) as adjust in hooks of UDFs. In order to show the process of fibrinolysis of clot and the results, the profiles of $\varepsilon$ and $C_{\alpha}$ were added into UDFs.

The mesh quality of this model is obtained in the CFD simulation, the minimum body size of the global grid is $1.8456 e-3 \mathrm{~mm}$, and the computational grid cells are 274958 .

For parameters, we introduce the effect of AP and set the concentration of AP to be $1.0 \mu \mathrm{M}$ in free phase [16]. For other initial conditions, fibrin density $\left(\rho_{\text {fibre }}\right)$ is $0.28 \mathrm{mg} / \mathrm{ml}$ [16] and fibrin radius $\left(R_{\mathrm{f} 0}\right)$ is $250 \mathrm{~nm}$ [28]. The concentration of PLG in the free phase and bound phase is set as $21.65 \mu \mathrm{M}$ and $34.9 \mu \mathrm{M}$, respectively [16]. For the proportion of drug, the concentration of PLG in drug is $2 \mu \mathrm{M}$ same as concentration of PLG in plasma. And the plasma concentration of tPA is low as $70 \mathrm{nM}$; thus, the variety of tPA concentration in drug can make influence for dissolution efficiency. This model added these parameters as initialization of hooks of UDFs.

For boundary conditions, the pressure of vessel inlet is $10 \mathrm{~Pa}$ and the pressure of vessel outlet is $0 \mathrm{~Pa}$ [29]. In order to get the influence of injection velocity, it varies from $10 \mathrm{~mm} / \mathrm{s}$ to $100 \mathrm{~mm} / \mathrm{s}$. In order to simplify the model, nonpermeable and nonslip walls are introduced.

Based on the simulation method which uses transient analysis to calculate the flow at different times, this study sets several simulations to investigate the effect of tPA concentration, injection velocity, injection position, and size of clot.

For tPA concentration, this study sets tPA concentration of the drug between $10 \mathrm{nM}$ and $100 \mathrm{nM}$ to test and obtain the influence of tPA concentration in the drug. As a basic set, the velocity of injection is $40 \mathrm{~mm} / \mathrm{s}$, the length of clot is $0.1 \mathrm{~mm}$, and the injection site (the distance from clot to outlet of microneedle) is $0.3 \mathrm{~mm}$ in front of the thrombus.

For injection velocity, it changes from $10 \mathrm{~mm} / \mathrm{s}$ to $100 \mathrm{~mm} / \mathrm{s}$. Other conditions remain unchanged, length of clot is $0.1 \mathrm{~mm}$, and the injection site is $0.3 \mathrm{~mm}$ in front of the thrombus. However, tPA concentration for injection velocity is constant for high efficiency which is illustrated from the experiment for tPA concentration.

For injection position, it can be divided into external and internal clot. In actual surgery, the position of injection is in front of clot; thus, we set the injection site to $0.1 \mathrm{~mm}$, $0.2 \mathrm{~mm}, 0.3 \mathrm{~mm}, 0.4 \mathrm{~mm}$, and $0.5 \mathrm{~mm}$ in front of clot, respectively. Additionally, due to the high difficulty and immaturity for cannulation surgery, inadvertent operation may occur which leads cannula into the thrombus. The event of the microneedle being injected inside of the clot was taken into consideration, and three measurements of injection depth along $X$-axis were used for experiment: $0.025 \mathrm{~mm}$, $0.05 \mathrm{~mm}$, and $0.075 \mathrm{~mm}$. The effects of different injection positions are simulated under the situation where the concentration of tPA stays a constant value chosen from the tPA concentration experiment, velocity of injection is determined by the injection velocity experiment, and length of clot is $0.1 \mathrm{~mm}$.

For the effects of clot size, the chosen sizes are $0.1 \mathrm{~mm}$, $0.2 \mathrm{~mm}, 0.3 \mathrm{~mm}, 0.4 \mathrm{~mm}, 0.5 \mathrm{~mm}$, and $0.6 \mathrm{~mm}$. This part of work records the fibrinolytic time over different lengths of clot with various tPA concentration and tPA concentration.

\section{Results}

3.1. Validation of the Method. For the sake of validation, this study repeats simulations to replicate the computational and experimental conditions from literatures [22, 27]. The results are consistent with those simulated in the literature [27] with $k_{\mathrm{M}}$ and $k_{2}$ obtained from Table 1 . Using the results obtained by Blinc et al. [25], we replicated the experimental conditions from literatures [22] and assumed that the kinetic parameters for plasminogen activation were $\mathrm{kM}=2.42 \mu \mathrm{M}$ and $k 2=0.22 \mathrm{~s}^{-1}$ [22]. The difference between our results and numerical results of the literature [27] and experimental results of the literature [22] is $13 \%$ and $7 \%$, respectively, as shown in Figure 4(a). Figure 4 shows the time taken when the clot is dissolved up to $95 \%$ and deviation of comparisons between simulation results and experimental results. Due to a low deviation rate in Figure 4(b), the accuracy of this model can be verified.

3.2. Effect of tPA Concentration. The drug tPA concentration was set as $10 \mathrm{nM}$ to $100 \mathrm{nM}$ to the effects of tPA concentration. Figure 5 shows lysis time over variations of tPA concentration, which explores the influence of tPA 


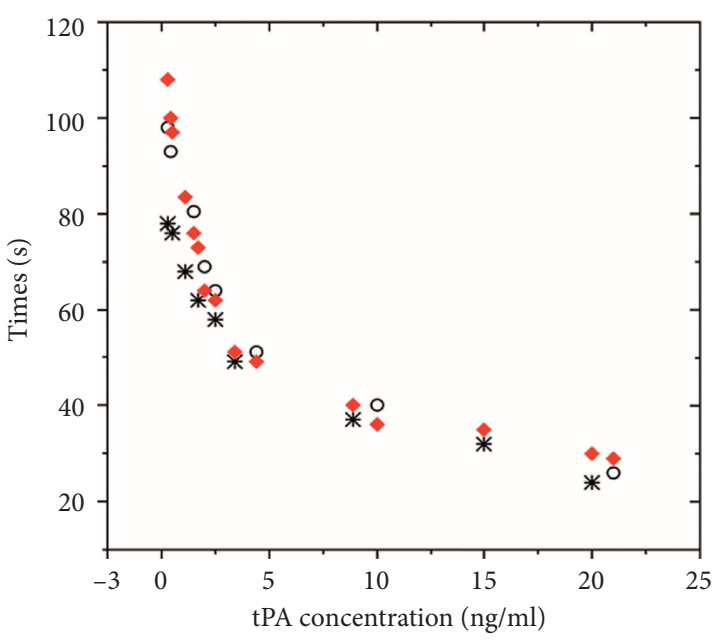

(a)

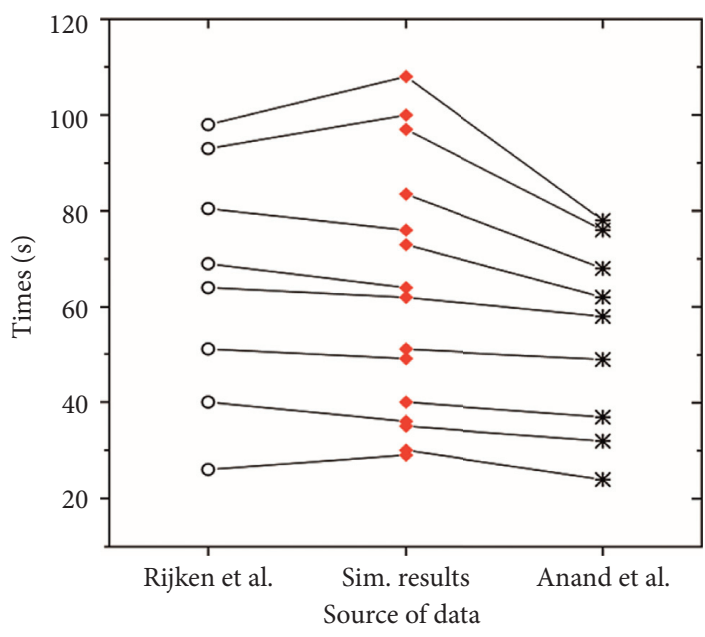

(b)

Figure 4: (a) The lytic time of simulation results and previous experiments with the condition of 95\% thrombolysis. (b) The comparison of simulation results with experimental results, and low deviation explores high similarity.

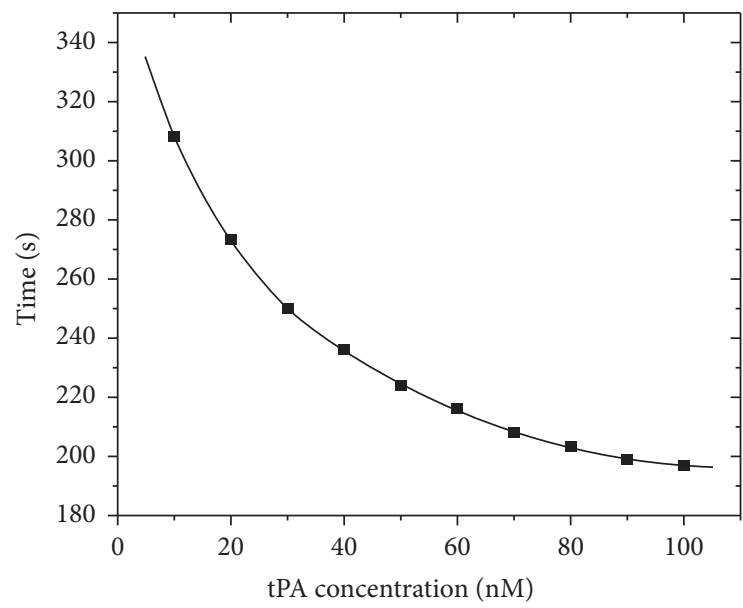

Figure 5: Lysis time over tPA concentration.

concentration in drug. The changing trend of nodes is narrow with a sharp peak that rapidly falls off and the profile becomes wider over tPA concentration. We analyzed the data to establish the polynomial fitting curve which can be written as follows:

$$
T_{\mathrm{tPA}}=a_{1} I_{\mathrm{tPA}}+a_{2} I_{\mathrm{tPA}}^{2}+a_{3} I_{\mathrm{tPA}}^{3}+k_{1}
$$

$a_{1}, a_{2}, a_{3}$, and $k_{1}$ and other values can be obtained from Table 2, $T_{\mathrm{tPA}}$ is fibrinolytic time, $I_{\mathrm{tPA}}$ is tPA concentration of injection, and its R-squared is 0.99784 which means fitting well. This curve is presented in Figure 5 showing a decrease in time for lysis when tPA concentration increased from $0 \mathrm{nM}$ to $50 \mathrm{nM}$. However, the rate of change of lysis time slows down gradually when tPA concentration reaches over $50 \mathrm{nM}$. When tPA concentration increased from $0 \mathrm{nM}$ to $50 \mathrm{nM}$, the 1st derivative of this curve increases rapidly. However, then it increases slowly with concentration reaching over $50 \mathrm{nM}$, which clearly demonstrates that the
TABle 2: Parameter.

\begin{tabular}{lc}
\hline Symbol & Quantity \\
\hline$a_{1}$ & $-4.40845 \pm 4.00535$ \\
$a_{2}$ & $0.04885 \pm 0.00619$ \\
$a_{3}$ & $-1.97552 e-4 \pm 3.71336 e-5$ \\
$k_{1}$ & $345.56667 \pm 4.00535$ \\
Residual sum of squares & 25.55501 \\
$R$-squared & 0.99784 \\
$R$ square after adjustment & 0.99676 \\
\hline
\end{tabular}

most efficient value of tPA concentration is around $50 \mathrm{nM}$ (for thrombolysis of $230 \mathrm{~s}$ ).

Figure 6 shows the fibrinolysis contours of voidage under the condition, where tPA concentration and injection velocity are $50 \mathrm{nM}$ and $40 \mathrm{~mm} / \mathrm{s}$, respectively. Figure 6 show the transversion of thrombolysis contours for the $0.1 \mathrm{~mm}$ clot where the initial voidage is about 0.98 which means the intact clot and ultimate voidage is 1.0 which means the completed lysis. The time taken for clot lysis to complete with tPA concentration of $50 \mathrm{nM}$ recorded at around $236 \mathrm{~s}$, as shown in Figure 6, where lysis was found to start at the center in front of clot and formed a conical space allowing thrombolysis to undertake gradually. The result matches the simulation data from [21] to further prove accuracy.

3.3. Effect of Injection Velocity. Figure 7 shows lysis time over various injection velocity with tPA concentration of $50 \mathrm{nM}$ obtained from tPA concentration stimulations. The time profile falls off rapidly and gets wider, and lysis time for clot lysis to complete changed from $273 \mathrm{~s}$ to $120 \mathrm{~s}$ corresponding to $10 \mathrm{~mm} / \mathrm{s}$ to $60 \mathrm{~mm} / \mathrm{s}$. Then, the various scale of dissolution time is reduced from $120 \mathrm{~s}$ to $107 \mathrm{~s}$ with injection velocity of $60 \mathrm{~mm} / \mathrm{s}$ to $100 \mathrm{~mm} / \mathrm{s}$. The data of simulation and the polynomial fitting curve are presented in Figure 6. And the polynomial fitting equation can be written as follows: 


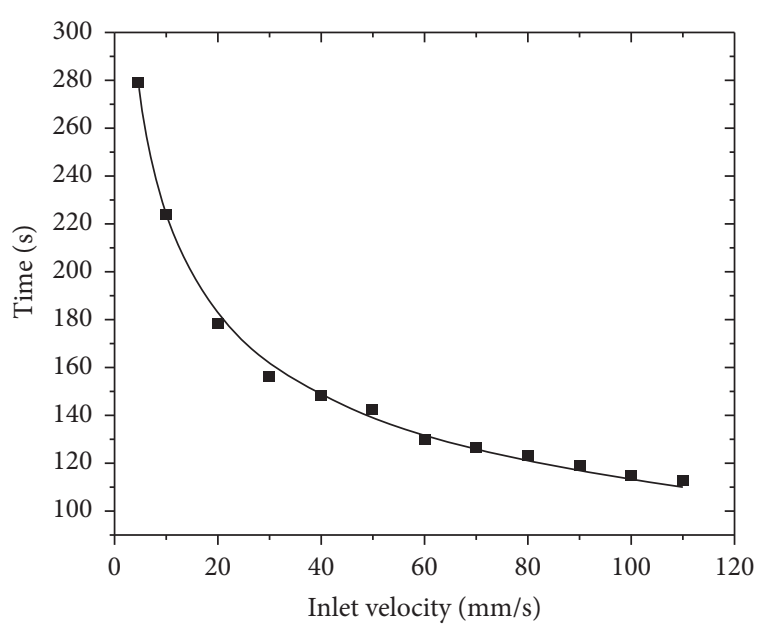

Figure 6: Lysis time over injection velocity.

$$
T_{\text {vel }=b_{1} v+b_{2} v^{2}+b_{3} v^{3}+b_{4} v^{4}+b_{5} v^{5}+b_{6} v^{6}+k_{2}},
$$

where $T_{\text {vel }}$ is fibrinolytic time over injection velocity, vis injection velocity, its $R$-squared is 0.99914 , which means fitting well, and other parameters are shown in Table 3. The 1st derivative of this curve increased rapidly from to $10 \mathrm{~mm} /$ $\mathrm{s}$ to $60 \mathrm{~mm} / \mathrm{s}$ and slowly from $60 \mathrm{~mm} / \mathrm{s}$ to $100 \mathrm{~mm} / \mathrm{s}$. This profile revealed that the most efficient value of injection velocity is around $60 \mathrm{~mm} / \mathrm{s}$ (for thrombolysis of $120 \mathrm{~s}$ ).

In addition, Figure 8 shows the flow vectors superimposed on velocity streamlines from microneedle inlet for different velocities effecting the time of lysis. The velocity of $20 \mathrm{~mm} / \mathrm{s}$ leads to low and stable drug flow showing in Figures 8 (a) and $8(\mathrm{~b})$. A higher velocity of $50 \mathrm{~mm} / \mathrm{s}$ causes the drug to spurt out from cannula and fill with vessel to start thrombolysis towards the front surface of clot with higher lysis velocity in Figures 8(c) and 8(d). Figures 8(e) and 8 (f) explicitly show that drug spurts out from microneedle and causes partial regurgitation by velocity of $100 \mathrm{~mm} / \mathrm{s}$.

3.4. Effect of Injection Position. Figures 9 and 10 show the thrombolysis time over the thrombolysis ratio for different positions. In Figure 9, the trend of three profiles is similar where time increases slowly when the thrombolysis ratio is under 0.6, and the increase of time becomes exponential when the thrombolysis ratio is higher than 0.6 . The ratio cannot get to $100 \%$, which means that thrombolysis is incomplete when the distance between the microneedle inlet and the front of the clot is under $0.2 \mathrm{~mm}$. In order to explain this phenomenon, Figure 11 shows the tPA concentration contours for the thrombolytic process when the microneedle is $0.2 \mathrm{~mm}$ in front of the clot. In Figure 10, the increment of time increases rapidly when the thrombolysis ratio is lower than 0.1 ; then, the profiles get wider and increase sharply again until thrombolysis ratio reaches 1.0 exploring dissolution of clot completely. Thrombolysis can be completed with the distance of $0.3 \mathrm{~mm}$ and longer distance, and the thrombolysis time is the minimum with the distance of $0.4 \mathrm{~mm}$.
Table 4 shows the clot lysis time for internal cannulation. With the increasing length of insertion, the percentage of dissolution drops with shorter time. Figure 12 shows the voidage contours of $0.025 \mathrm{~mm}$ inside the clot and $0.1 \mathrm{~mm}$, $0.2 \mathrm{~mm}$, and $0.3 \mathrm{~mm}$ in the front of clot to present the evolution of clot lysis. Figure 12 shows clot lysis starts from the port of microneedle and the area of dissolution changes from a cylinder to a funnel to the end of thrombolysis and most of the thrombus around the needle cannot be dissolved. Figures 12(b) and 12(c) show the incomplete thrombolysis in the end, while floccules of clot.

3.5. Effect of Clot Size. Based on the results of condition simulations and injection mode simulations for thrombolysis, this study obtained more efficient values of $50 \mathrm{nM}$ for tPA concentration, $60 \mathrm{~mm} / \mathrm{s}$ for injection velocity, and $0.4 \mathrm{~mm}$ for distance in the front of the clot. In Figure 6, the thrombolysis process is explored by lysis contours for different sizes of $0.1 \mathrm{~mm}, 0.2 \mathrm{~mm}$, and $0.3 \mathrm{~mm}$. Figure 13 shows thrombolytic time gets longer as tPA concentration gets higher with any clot size between $0.1 \mathrm{~mm}$ and $0.6 \mathrm{~mm}$ under the condition, where injection velocity is $60 \mathrm{~mm} / \mathrm{s}$ and injection position is $0.4 \mathrm{~mm}$ in front of clot. Figure 14 shows the simulation results of thrombolysis time gets longer over different injection velocities under condition of tPA concentration of $50 \mathrm{nM}$ and injection position of $0.4 \mathrm{~mm}$.

\section{Discussion}

In this paper, we obtained the results of four groups of simulations showing the influence of tPA concentration, injection velocity, injection position, and various clot sizes on thrombolysis. From these results, we can explore how the thrombolytic completion and time vary with the critical parameters, and these change rules can be used as a reference for surgery.

Proper tPA concentration is important because of the potential retinal toxicity of high concentration [31]. The curve in Figure 5 can be used to obtain determination rules of drug concentration to be applied to robot-assisted cannulation and is similar to the results in Sriram Anand's work, where they simulated tPA concentration of from $0.1 \mathrm{ng} / \mathrm{ml}(0.0014 \mathrm{nM})$ to $100 \mathrm{ng} / \mathrm{ml}(1.4 \mathrm{nM})$ with fibrin concentration of $5.88 \mu \mathrm{m}$ [27]. The reasons of efficient tPA concentration of $50 \mathrm{nM}$ are the strong influence of concentration in the low range $(10 \mathrm{nM}-50 \mathrm{nM})$ and decreasing derivative of lysis time changing. This prediction is consistent with the results from Mark W and Johnson MD' work [30], which revealed proper tPA concentration performed well in progressive intraoperative clot dissolution, while higher concentration failed to make efficient thrombolysis.

To investigate thoroughly the effect of inlet velocity, we compared the clot lysis process of $20 \mathrm{~mm} / \mathrm{s}, 50 \mathrm{~mm} / \mathrm{s}$, and $80 \mathrm{~mm} / \mathrm{s}$ by velocity streamlines displaying in Figure 8 which clearly indicates the reasons for different thrombolysis time periods. Firstly, the disappeared jet flow for velocity of 


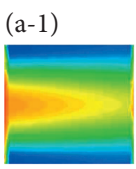

(a-2)

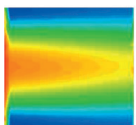

(a-3)

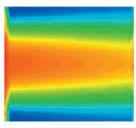

(a-4)

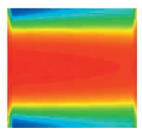

(a) (b-1)

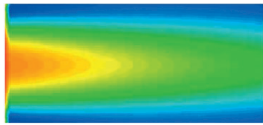

(b-2)

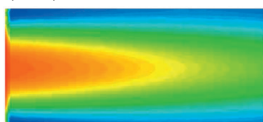

(b-3)

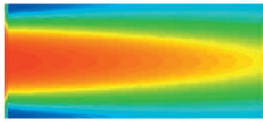

(b-4)

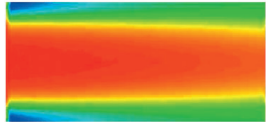

(b)
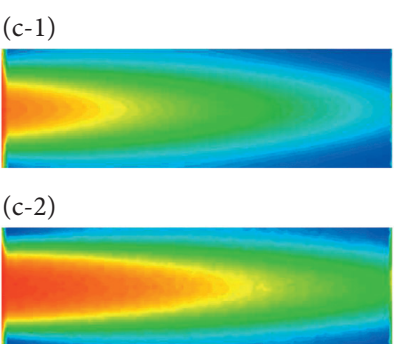

(c-3)

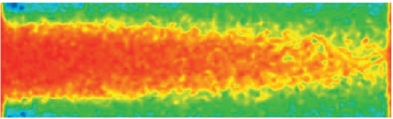

(c-4)

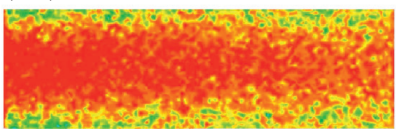

(c)

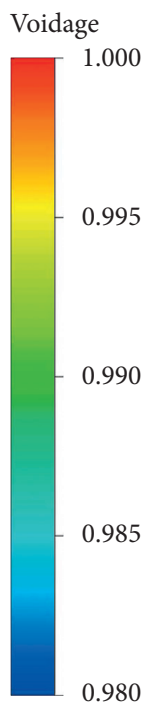

0.980

Figure 7: Voidage of lytic process for clot length of (a) $0.1 \mathrm{~mm}$, (b) $0.2 \mathrm{~mm}$, and (c) $0.3 \mathrm{~mm}$. The range of voidage is from 0.980 to 1.000 . The red regions denote areas of complete dissolution, while blue regions mean that the dissolution has not started yet.

TABle 3: Parameter.

\begin{tabular}{lc}
\hline Symbol & Quantity \\
\hline$b_{1}$ & $-21.0712 \pm 1.72499$ \\
$b_{2}$ & $0.90982 \pm 0.12345$ \\
$b_{3}$ & $-0.02105 \pm 0.00391$ \\
$b_{4}$ & $2.62314 e-4 \pm 6.09204 e-5$ \\
$b_{5}$ & $-1.66236 e-6 \pm 4.57943 e-7$ \\
$b_{6}$ & $4.19977 e-9 \pm 1.3248 e-9$ \\
$k_{1}$ & $364.30138 \pm 7.49007$ \\
Residual sum of squares & 24.37624 \\
$R$-squared & 0.99914 \\
$R$ square after adjustment & 0.9981 \\
\hline
\end{tabular}

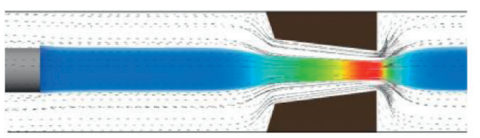

(a)

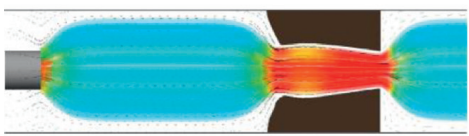

(c)

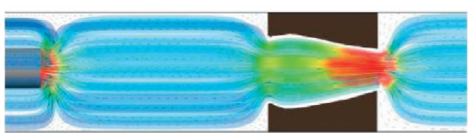

(e)

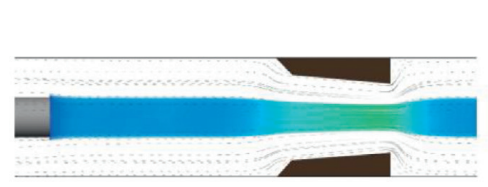

(b)

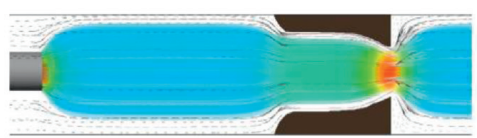

(d)

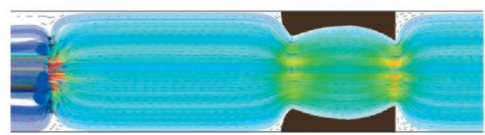

(f)

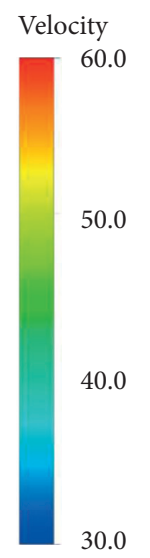

$\left(\mathrm{mm} \mathrm{s}^{-1}\right)$

FIGURE 8: The flow vectors superimposed on velocity streamlines from microneedle inlet for different injection velocity of $20 \mathrm{~mm} / \mathrm{s}$ ( $\mathrm{a}, \mathrm{b}$ ), $50 \mathrm{~mm} / \mathrm{s}(\mathrm{c}, \mathrm{d})$, and $80 \mathrm{~mm} / \mathrm{s}(\mathrm{e}, \mathrm{f})$. The red regions show areas of velocity of $60.0 \mathrm{~mm} / \mathrm{s}$ or higher, while the blue regions denote areas of velocity of $30 \mathrm{~mm} / \mathrm{s}$ or lower.

$20 \mathrm{~mm} / \mathrm{s}$ causes longer clot lysis time. However, the vessel can be filled with drug at the proper velocity of $50 \mathrm{~mm} / \mathrm{s}$, which leads to efficient thrombolysis. This illustrates that appropriately setting the velocity at $60 \mathrm{~mm} / \mathrm{s}$ is more efficient than having a higher or lower velocity [16]. In Tameesh's study [32], a high injection flow rate of $0.05 \mathrm{ml} / \mathrm{min}$ causes 


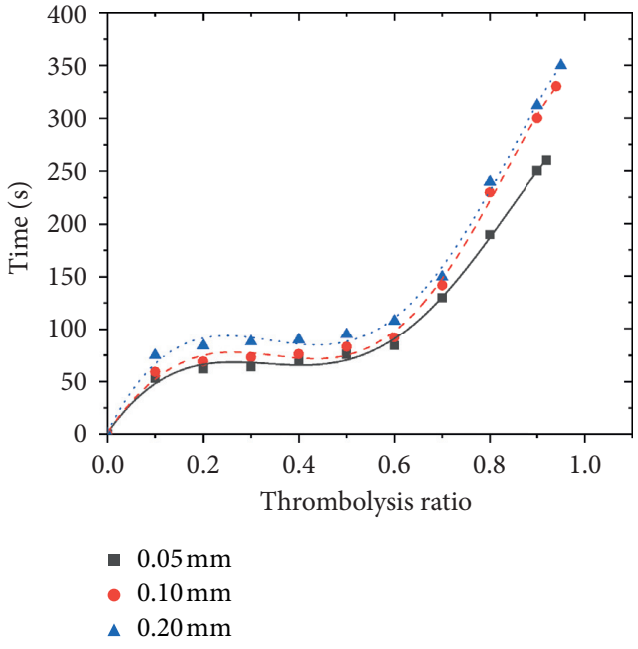

Figure 9: Thrombolysis time for different positions of $0.05 \mathrm{~mm}$, $0.10 \mathrm{~mm}$, and $0.20 \mathrm{~mm}$. Thrombolysis ratio of 1.0 denotes complete dissolution.

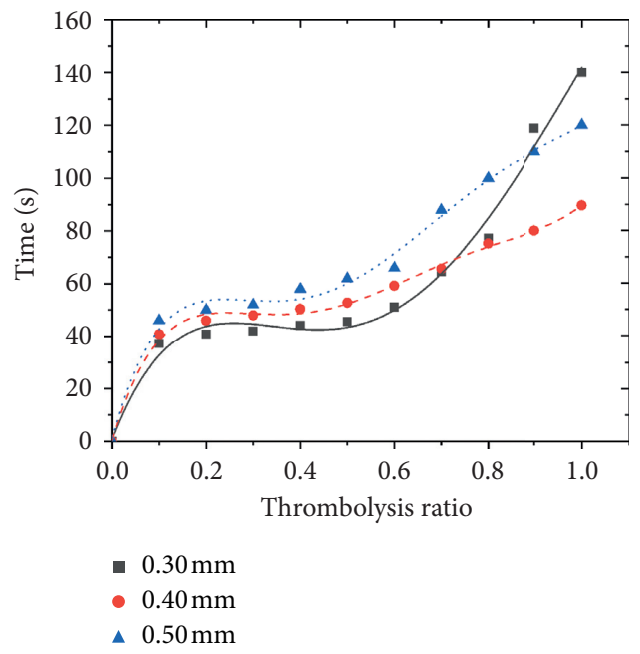

Figure 10: Thrombolysis time for different positions of $0.30 \mathrm{~mm}$, $0.40 \mathrm{~mm}$, and $0.50 \mathrm{~mm}$. Thrombolysis ratio of 1.0 denotes complete dissolution.

inefficient thrombolysis. At last, the high velocity of $80 \mathrm{~mm} / \mathrm{s}$ causes reflux at the back of clot where the development of thrombolysis slows down. These results of injection velocity can be used to get determination rules of injection dosage and velocity of drug to reduce drug waste and prevent drug reflux in the process of thrombolysis.

For the injection position, it can be seen that thrombolysis efficiency is good when the thrombolytic ratio is about 0.6 with distance of lower than $0.2 \mathrm{~mm}$. With the increase of thrombolytic ratio, the thrombolytic time increases rapidly, and the thrombolytic process cannot be completed Figure 9. We can get the reason from Figure 11, which appears that the drug cannot penetrate into the thrombus on both sides of the blood vessel wall and flows out with the flow of blood after the clot is dissolved incompletely. The minimum thrombolytic time occurred around the distance of $0.4 \mathrm{~mm}$ because there are floccules formed in the end of thrombolytic process with distance of $0.3 \mathrm{~mm}$ or longer in Figure 12; thus, the undissolved clot can be washed away by blood flow [33]. Under the current conditions, we can get the position of the highest efficiency is between $0.3 \mathrm{~mm}$ and $0.5 \mathrm{~mm}$, which conforms with the results in the literature which illustrates that the best location for cannulation was directly proximal to the occlusion [7].

It can be concluded that the thrombolytic effect is not good when the microneedle is inserted into the thrombus. With low injection velocity, the drug cannot reach the thrombus on the left side and near the vessel wall. Even if the injection velocity increases, we cannot dissolve the thrombus near the blood vessel wall. Because the drug flows out directly with the blood stream after the thrombus on the right side of the micro-needle has been dissolved as Figure 12 shown. Avoiding thrombus insertion, the operation is easier to perform and the blood vessel is avoided to be damaged.

For the simulation results of different sizes of clot with various tPA concentration and injection velocity, we can conclude that, as injection velocity and drug concentration increased, thrombolytic efficiency increased extremely with a specific clot size. When injection velocity or drug concentration goes beyond a range, however, the influence is gradually diminished. The result of tPA concentration are very similar to those described in Hauptmann and Glusa's work [24]. In addition, the trend in change of fibrinolytic time for low injection velocity consists with the results of Piebalgs and Xu's work [17]. Because of this, we can obtain that the higher efficient range of tPA concentration and injection velocity are from $20 \mathrm{nM}$ to $70 \mathrm{nM}$ and from $40 \mathrm{~mm} /$ $\mathrm{s}$ to $60 \mathrm{~mm} / \mathrm{s}$, respectively. Proper values need to be determined in the simulation database according to the specific thrombus size.

By data analysis, we can get change rules of effects of drug concentration, injection velocity, and injection position on clot lysis. In the process of robot-assisted surgery, Visual Recognition System can obtain the size and position of clot and the environment of injection with deep learning. On the basis of rules and size of clot, optimal tPA concentration and injection velocity can be determined to be fed into the cannulation operating system to increase the efficiency and success of surgery. In addition, cannulation will be performed in a reasonable position in terms of the size of clot to avoid the haemorrhage reaction [7]. This method can guide the catheter tip accurately to the surface of the vein and avoid inserting into clot to perform surgery successfully.

In this study, the simulation method has a number of limitations. This method simplifies the physical model where it ignores the changes of thrombus shape and vessel diameter and idealizes the injection position of the microneedle. Because of this, the convection of blood and drugs may be some deviation from the actual situation, which can affect fibrinolytic time to a certain extent. Constant vessel diameter also leads to limitations of simulation database.

In the future, we plan to supplement the effect of vessel diameter and geometry of clot on thrombolysis to make the simulation database more comprehensive. And these results will be used to assist the performance of cannulation. 


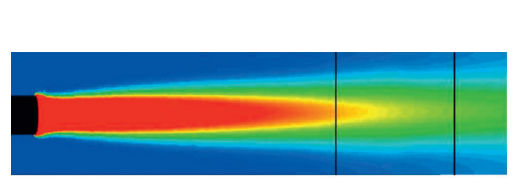

(a)

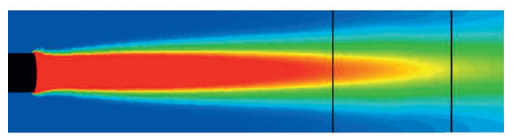

(c)

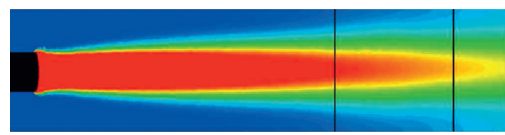

(e)

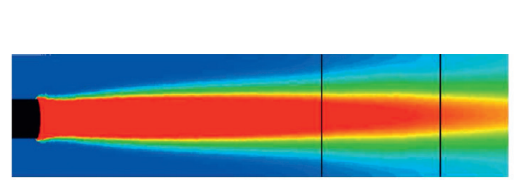

(b)

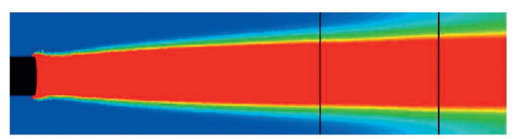

(d)

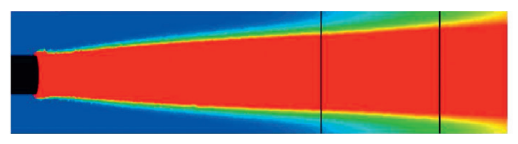

(f)
Concentration

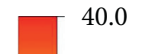

30.0

20.0

10.0

0.0

FIgURE 11: The tPA concentration contours for the thrombolytic process of external position of $0.2 \mathrm{~mm}$. The concentration of (a)-(f) changes over time. Blue area means low tPA concentration and red area means high concentration.

TABLE 4: Thrombolytic time of insertion.

\begin{tabular}{lcc}
\hline Length of insertion $(\mathrm{mm})$ & Percentage of dissolution & Time $(\mathrm{s})$ \\
\hline 0.025 & 0.85 & 687 \\
0.05 & 0.67 & 550 \\
0.075 & 0.31 & 371 \\
\hline
\end{tabular}

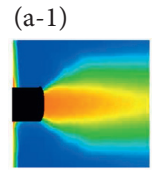

$(\mathrm{a}-2)$

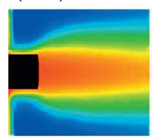

$(\mathrm{a}-3)$

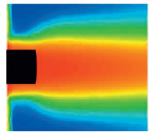

$(\mathrm{a}-4)$

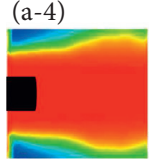

(a)

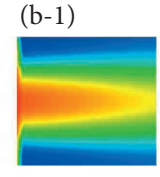

(b-2)

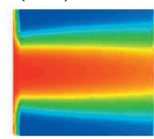

(b-3)

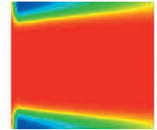

(b-4)

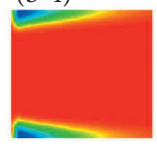

(b)

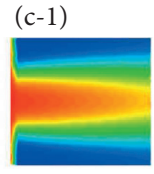

(c-2)

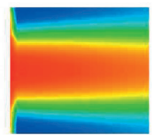

(c-3)

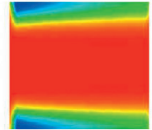

(c-4)

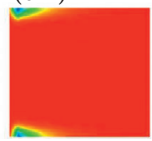

(c)

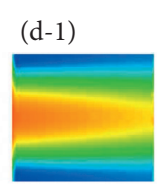

(d-2)

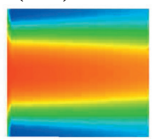

(d-3)

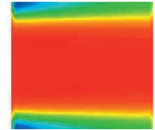

(d-4)

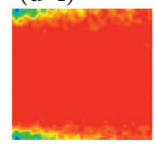

(d)

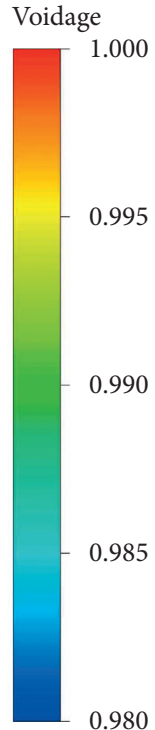

0.980

FIgURE 12: The voidage contours of $0.025 \mathrm{~mm}$ inside the clot (a), and $0.1 \mathrm{~mm}(\mathrm{~b}), 0.2 \mathrm{~mm}$ (c), and $0.3 \mathrm{~mm}$ (d) in the front of the clot to present the evolution of clot lysis. 


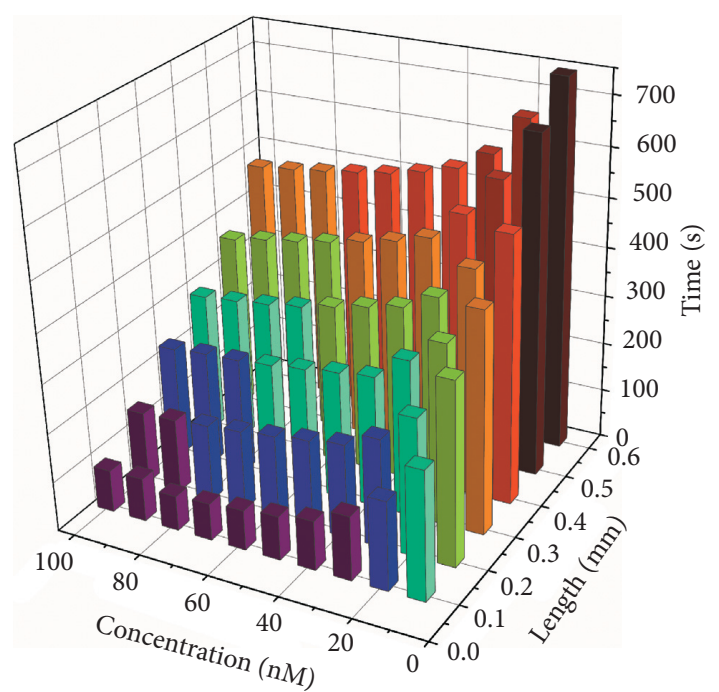

Figure 13: The variation of lysis time over different tPA concentrations and various clot sizes.

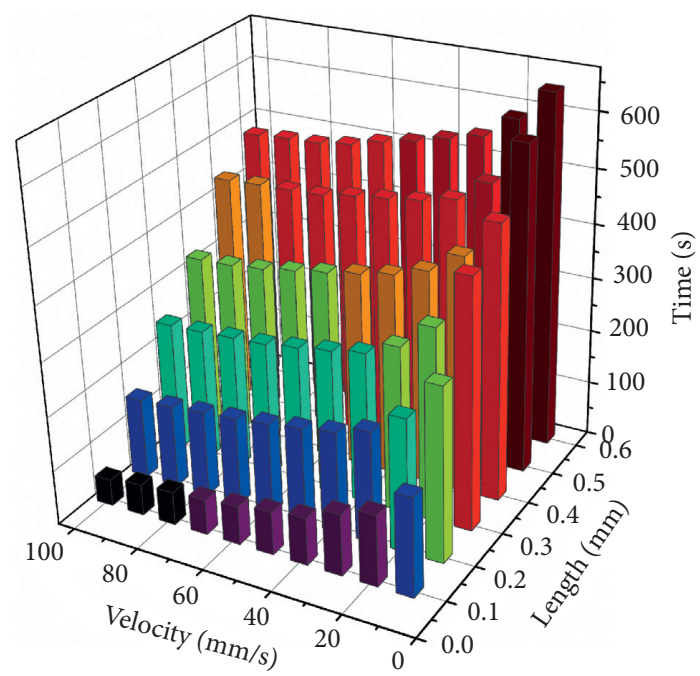

Figure 14: The variation of lysis time over different injection velocities and various clot sizes.

\section{Conclusions}

Because of few cases of robotic intubation surgery, there is a lack of data on the effects of drug concentration, injection velocity, injection location, and clot size on fibrinolytic efficacy. The accuracy of the multiphysics continuum model established by finite analysis element is verified by comparing with literatures. In this paper, the data are obtained by simulation of cannulation operation where the influence of microneedle is considered. For clot length of $0.1 \mathrm{~mm}$ to $0.6 \mathrm{~mm}$, the data indicates that the optimal tPA concentration ranged from $20 \mathrm{nM}$ to $70 \mathrm{nM}$ and the optimal injection velocity ranged from $40 \mathrm{~mm} / \mathrm{s}$ to $60 \mathrm{~mm} / \mathrm{s}$, and the thrombolysis cannot be completed when the microneedle is inserted into the thrombus and the position of the highest efficiency is between $0.3 \mathrm{~mm}$ and $0.5 \mathrm{~mm}$ under conditions in this paper. In the future, we will consider the influence of vessel diameter to supplement the database and apply the data in this paper to robotic-assisted intubation surgery.

\section{Data Availability}

All data generated or used during the study appear in the submitted article.

\section{Conflicts of Interest}

The authors declare that they have no conflicts of interest.

\section{Authors' Contributions}

Shunlei Li and Jiawen Pan contributed equally to this study.

\section{References}

[1] S. Rogers, R. L. Mcintosh, N. Cheung et al., "The prevalence of retinal vein occlusion: pooled data from population studies from the United States, Europe, asia, and Australia," Ophthalmology, vol. 117, no. 2, pp. 313-319, 2010.

[2] J. Rehak and M. Rehak, "Branch retinal vein occlusion: pathogenesis, visual prognosis, and treatment modalities," Current Eye Research, vol. 33, no. 2, pp. 111-131, 2008.

[3] J. C. Clarhson, "Natural history and clinical management of central retinal vein occlusion," A.M.A. Archives of Ophthalmology, vol. 115, no. 4, pp. 486-491, 1997.

[4] P. Jefferies, R. Clemett, and T. Day, "An anatomical study of retinal arteriovenous crossings and their role in the pathogenesis of retinal branch vein occlusions," Clinical \& Experimental Ophthalmology, vol. 21, no. 4, pp. 213-217, 2010.

[5] P. Kolar, "Risk factors for central and branch retinal vein occlusion: a meta-analysis of published clinical data," Journal of Ophthalmology, vol. 2014, no. 7, Article ID 724780, 2014.

[6] N. Feltgen, D. Schmidt, and L. Hansen, "Occlusion of the Retinal artery," Der Ophthalmologe: Zeitschrift der Deutschen Ophthalmologischen Gesellschaft, vol. 100, no. 8, pp. 651-665, 2003.

[7] M. D. D. Smet, C. M. Thijs, Meenink et al., "Retinal vein cannulation with prolonged infusion of tissue plasminogen activator ( $t-P A)$ for the treatment of experimental retinal vein occlusion in dogsic assisted cannulation of occluded retinal veins," PLoS One, vol. 11, no. 9, Article ID e0162037, 2016.

[8] R. P. Finger, S. S. Wickremasinghe, P. N. Baird, and R. H. Guymer, "Predictors of anti-VEGF treatment response in neovascular age-related macular degeneration," Survey of Ophthalmology, vol. 59, no. 1, pp. 1-18, 2014.

[9] I. Keiji, O. Kishiko, O. Sachiko et al., "Subthreshold micropulse photocoagulation for persistent macular Edema secondary to branch retinal vein occlusion including best-corrected visual acuity greater than 20/40," Journal of Ophthalmology, vol. 2014, pp. 1-10, 2014.

[10] K. A. Van Overdam, T. Missotten, and L. H. Spielberg, "Updated cannulation technique for tissue plasminogen activator injection into peripapillary retinal vein for central retinal vein occlusion," Acta Ophthalmologica, vol. 93, no. 8, pp. 739-744, 2015.

[11] R. Doolittle, "Fibrinogen and fibrin," Advances in Protein Chemistry, vol. 70, no. 6, pp. 247-299, 2005.

[12] X. Fang, T. W. Jie, and T. Feng, "An industrial micro-defect diagnosis system via intelligent segmentation region," Sensors, vol. 19, no. 11, p. 2636, 2019. 
[13] M. D. D. Smet, J. M. Stassen, T. C. M. Meenink et al., "Release of experimental retinal vein occlusions by direct intraluminal injection of ocriplasmin," British Journal of Ophthalmology, vol. 100, no. 12, p. 1742, 2016.

[14] K. Willekens, A. Gijbels, L. Schoevaerdts et al., "Robotassisted retinal vein cannulation in an in vivo porcine retinal vein occlusion model," Acta Ophthalmologica, vol. 95, no. 3, pp. 270-275, 2016.

[15] A. Gijbels, J. Smits, L. Schoevaerdts et al., "In-human robotassisted retinal vein cannulation, a world first," Annals of Biomedical Engineering, vol. 46, no. 10, pp. 1676-1685, 2018.

[16] S. L. Diamond and S. Anand, "Inner clot diffusion and permeation during fibrinolysis," Biophysical Journal, vol. 65, no. 6, pp. 2622-2643, 1994.

[17] A. Piebalgs and X. Y. Xu, "Towards a multi-physics modelling framework for thrombolysis under the influence of blood flow," Journal of the Royal Society Interface, vol. 12, no. 113, 2015.

[18] Y. Wu, H. Zhao, C. Zhang, L. Wang, and J. Han, "Optimization analysis of structure parameters of steam ejector based on CFD and orthogonal test," Energy, vol. 151, pp. P79-P93, 2018.

[19] D. M. Wootton, A. S. Popel, and B. Rita Alevriadou, "An experimental and theoretical study on the dissolution of mural fibrin clots by tissue-type plasminogen activator," Biotechnology and Bioengineering, vol. 77, no. 4, pp. 405-419, 2002.

[20] B. E. Bannish, J. P. Keener, M. Woodbury, J. W. Weisel, and A. L. Fogelson, "Modelling fibrinolysis: 1D continuum models," Mathematical Medicine and Biology, vol. 31, no. 1, p. 45, 2014.

[21] B. E. Bannish, J. P. Keener, and A. L. Fogelson, "Modelling fibrinolysis: a 3D stochastic multiscale model," Mathematical Medicine and Biology, vol. 31, no. 1, p. 17, 2012.

[22] D. C. Rijken, M. Hoylaerts, and D. Collen, "Fibrinolytic properties of one-chain and two-chain human extrinsic (Tissue-type) plasminogen activator," Journal of Biological Chemistry, vol. 257, no. 6, pp. 2920-2925, 1982.

[23] M. J. Dumskyj, S. J. Aldington, C. J. Doré, E. M. Kohner et al., "The accurate assessment of changes in retinal vessel diameter using multiple frame electrocardiograph synchronised fundus photography," Current Eye Research, vol. 15, no. 6, pp. 625-632, 1996.

[24] J. Hauptmann and E. Glusa, "Differential effects of staphylokinase, streptokinase and tissue-type plasminogen activator on the lysis of retracted human plasma clots and fibrinolytic plasma parameters in vitro," Blood Coagulation \& Fibrinolysis, vol. 6, no. 6, pp. 579-586, 1995.

[25] N. A. Booth, A. J. Simpson, A. Croll, B. Bennett, and I. R. MacGregor, "Plasminogen activator inhibitor (PAI-1) in plasma and platelets," British Journal of Haematology, vol. 70, no. 3, pp. 327-333, 1988.

[26] A. J. Tiefenbrunn, R. A. Graor, A. K. Robison, F. V. Lucas, A. Hotchkiss, and B. E. Sobel, "Pharmacodynamics of tissuetype plasminogen activator characterized by computerassisted simulation," Circulation, vol. 73, no. 6, pp. 1291-1299, 1986.

[27] S. Anand, J.-H. Wu, and S. L. Diamond, "Enzyme-mediated proteolysis of fibrous biopolymers: dissolution front movement in fibrin or collagen under conditions of diffusive or convective transport," Biotechnology and Bioengineering, vol. 48, no. 2, pp. 89-107, 1995.
[28] A. Blinc, J. Magdic, J. Fric et al., "Atomic force microscopy of fibrin networks and plasma clots during fibrinolysis," Fibrinolysis and Proteolysis, vol. 14, no. 5, pp. 0-299, 2000.

[29] J.-H. Wu, K. Siddiqui, and S. L. Diamond, "Transport phenomena and clot dissolving therapy: an experimental investigation of diffusion-controlled and permeation-enhanced fibrinolysis," Thrombosis and Haemostasis, vol. 72, no. 1, pp. 105-112, 1994.

[30] M. W. Johnson, K. R. Olsen, and E. Hernandez, "Tissue plasminogen activator thrombolysis during surgical evacuation of experimental subretinal hemorrhage," Ophthalmology, vol. 99, no. 4, pp. 515-521, 1992.

[31] A. Daruich, Parcq, Jérôme, K. Delaunay et al., "Retinal safety of intravitreal rtPA in healthy rats and under excitotoxic conditions," Molecular Vision, vol. 22, pp. 1332-1341, 2016.

[32] M. K. Tameesh, R. R. Lakhanpal, G. Y. Fujii et al., "Retinal vein cannulation with prolonged infusion of tissue plasminogen activator (t-PA) for the treatment of experimental retinal vein occlusion in dogs," American Journal of Ophthalmology, vol. 138, no. 5, pp. 829-839, 2004.

[33] J. Wu, X. Zhou, Y. Hu et al., "Video microscope recording of the dynamic course of thrombosis and thrombolysis of the retinal vein in rabbits," Retina, vol. 30, no. 6, pp. 966-970, 2010.

[34] B. Cheng, A. Li, and G. Shi, "A mathematical model for calculating the "brittleness-ductility" drop coefficient of sandstone in mining zones," Discrete Dynamics in Nature and Society, vol. 2020, Article ID 2621672, 7 pages, 2020. 